\title{
Residual Stress Measurement in a High Strength T Butt Weld Specimen by the Neutron Diffraction and Deep Hole Drilling Techniques
}

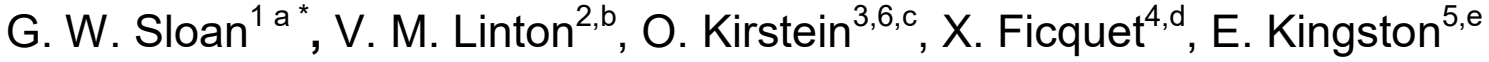 \\ ${ }^{1}$ School of Mechanical Engineering, University of Adelaide, North Terrace, Adelaide, Australia \\ ${ }^{2}$ Energy Pipelines CRC, Wollongong, Australia \\ ${ }^{3}$ Instrument Technology Division, European Spallation Source, Sweden \\ ${ }^{4}$ Veqter Ltd, Unicorn Business Park, Whitby Road, Bristol, BS4-4EX, UK \\ ${ }^{5}$ Veqter Ltd, Unicorn Business Park, Whitby Road, Bristol, BS4-4EX, UK \\ ${ }^{6}$ School of Mechanical Engineering, University of Newcastle, Callaghan, Newcastle, Australia \\ a *glen.sloan@bigpond.com.au, bvalerie.linton@epcrc.com.au, coliver.kirstein@esss.se, \\ xavier.ficquet@veqter.co.uk, ed.kingston@veqter.co.uk
}

\begin{abstract}
Keywords: Residual Stress, High Strength Steel, Quenched and Tempered, Neutron Diffraction, Deep Hole Drilling, T Butt Welds
\end{abstract}

\begin{abstract}
While defect assessment standards such as BS 7910 "Guide to methods of assessing the acceptability of flaws in metallic structures" [1] present residual stress profiles for T butt welds in thick sections they inherently assume that the weld pass placement strategy is such that the last weld pass is always on the base plate. There is limited information on what the residual stress profiles are if alternative pass placement strategies are used in high strength weldments and structures. Similarly there is limited information on what are the residual stress profiles under the body of the weld and the superimposition on the balancing residual stresses in the base plate away from the weld on preexisting residual stresses. This paper presents neutron strain scanning work on a high strength, curved base plate, double sided $\mathrm{T}$ Butt weld undertaken such that the pass placement in each layer progressed away from the base plate towards the abutting member to determine the potential of reduction in residual stresses at the base plate weld toes. Key results were validated by the Deep Hole Drilling Technique. The results obtained show that a major reduction in the magnitude of the residual stresses occurred at the weld toes of the base plate with a corresponding increase in the center of the weld. Weld toe through thickness profiles were reduced from those of BS 7910. Balancing residual stresses in the base plate were minimal. Discussion includes comparison to results obtained in similar test plates welded with a different pass placement strategy.
\end{abstract}

\section{Introduction}

Ring stiffened cylindrical structures for the offshore industry and naval platforms are usually fabricated from high strength steels with butt welds and $\mathrm{T}$ butt welds. The fabrication method involves manufacturing plate cans from thick plates with the longitudinal direction of initial rolling of the plate coinciding with that for shape rolling into a curve. Depending on the diameter 3 or more plates will be needed to be welded together to form a cylindrical can. The ring frames are also fabricated from high strength steel and are welded into the can via double sided $\mathrm{T}$ butt welds undertaken in the $1 \mathrm{G}$ down hand orientation. The same also applies for rigid bulkheads in the structures to resist buckling of the structures when subjected to sea pressure. These types of structures operate in environments where Stress Corrosion Cracking (SCC) is a frequent problem for

(c) (1) Content from this work may be used under the terms of the Creative Commons Attribution 3.0 license. Any further distribution of this work must maintain attribution to the author(s) and the title of the work, journal citation and DOI. Published under license by Materials Research Forum LLC. 
crack initiation in service as is Hydrogen Assisted Cold Cracking (HACC) during manufacture. In the case of naval platforms they are also subjected to a high stress low cycle fatigue regime throughout their service life. Therefore an understanding of residual stress profiles is essential for determining service life and structural integrity safety regimes for the welds and the structural components. While standards such as BS 7910 [1] present residual stress profiles for T butt welds they are limited to the weld toe region only and they assume that the last weld pass is on the base plate with the residual stress level being at tensile yield. No profiles are given for the region under the weld or in the base plates away from the welds and how much the existing profiles in the components are altered by adjacent welding. Hence there is a huge gap in knowledge of residual stress profiles throughout a fabricated ring stiffened cylindrical structure. In 2004 the University of Adelaide and its research partners, ISIS Rutherford Appleton Laboratories, Australian Nuclear Science and Testing Organization, ASC Pty Ltd, and the Commonwealth of Australia, implemented a program to address these gaps [2], [3]. The program was structured to look at the progressive build up or superimposition of residual stress profiles throughout the fabrication process for these types of structures and addressed both current and potential future weld pass placement strategies for the $T$ butt welds. The areas of primary interest were the base plate both beneath the weld and away from the weld region and also the weld. The material used throughout the entire program was BIS 812 EMA quenched and tempered plate with a minimum yield strength of $690 \mathrm{MPa}$ The primary method of determining the subsurface residual stress profiles was neutron diffraction (ND) as it was nondestructive and could be repeated with the destructive techniques Deep Hole Drilling (DHD), [4], and the Contouring technique being used to validate key profiles and also capture additional residual stress information where it was not possible to successfully neutron strain scan due to beam path length issues. This paper addresses the ND and DHD and incremental Deep Hole Drilling (iDHD), [4], results obtained on one of the $\mathrm{T}$ butt test pieces looking at the alternate weld pass placement strategy of progressing the weld passes in each layer from the base plate up to the web member. The results are extracted from [3].The welding for these test pieces was undertaken in the $1 \mathrm{G}$ orientation using the MMAW process at a heat input of $1.7 \mathrm{~kJ} / \mathrm{mm}$ with preheat of $120^{\circ} \mathrm{C}$ and outgassing at the same temperature to reduce risk of HACC. The weld consumable for the test pieces looking at this pass placement strategy was Atom Arc E120 consumables which produces welds of $690 \mathrm{MPa}$ yield strength or greater for this heat input.

\section{Experimental Methodology}

In order to maximize the longitudinal strain scan results in and under the weld this test piece was fabricated with a reduced number of layers by omitting the normal capping runs to give a slight taper as experience with the larger $T$ butt weld test piece of the series with full caps [3] had shown that with full caps the beam path lengths were excessive for successful longitudinal strain measurement in this region. The pass placement strategy for full cap and reduced cap are shown in Fig 1. This was preferred over cutting back the sample post welding to reduce the beam path length in the longitudinal direction due to concerns over relaxation of stresses. The test piece was fabricated with the lineup of longitudinal directions as shown in Fig 1. The test piece was fabricated in 2008 when the scanning table for the Kowari strain scanner was limited to $10 \mathrm{~kg}$ only and hence the test piece dimensions were limited in length to comply with this requirement while maintaining thickness of base plate and web member to other test pieces in the series [3]. The neutron strain scanning was undertaken on the Kowari strain scanner at the Bragg Institute, ANSTO, Sydney in 2010.

The plan for the neutron strain scanning is shown in Figure 2. As can be seen in this figure the through thickness scan lines concentrated on the area adjacent to and below the weld utilizing the reduced beam path lengths for longitudinal strain scans in both the weld and the zone immediately underneath the abutting web/weld. The methodology for undertaking DHD and iDHD residual stress measurements is given in [4] with the iDHD method being used in the plastic deformation regions of the heat affected zone and weld metal in addition to the DHD method, [4]. The subsequent locations 
for the DHD/iDHD tests are shown also in Fig 2. These were located at the $11 \mathrm{~mm}$ from the centerline of the web, so as to avoid the initial $1.5 \mathrm{~mm}$ gun drilled hole drill piece breaking if it was located at exactly the weld toe and the second set of DHD/iDHD measurements were undertaken at the centerline of the web. The general separation rule between DHD sites is $6 \mathrm{x}$ the diameter so as to avoid relaxation of residual stress at subsequent sites. As the two sets of DHD/iDHD measurements were undertaken several months apart the separation between the two DHD sites had to be $6 \times 5 \mathrm{~mm}$, i.e. $30 \mathrm{~mm}$, as the diameters of the final EDM core is $5 \mathrm{~mm}$, and therefore the second DHD site was centered further along the weld.
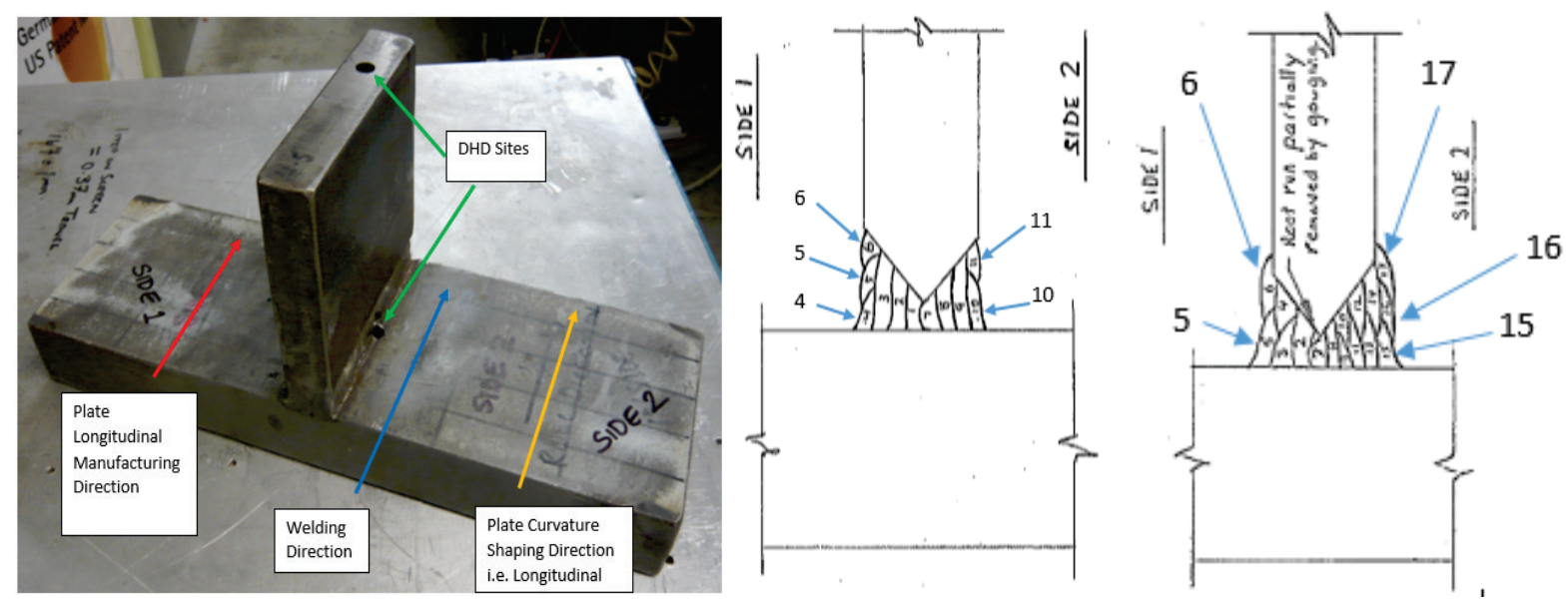

Fig 1 showing a) the cumulative line up of the longitudinal directions of the main manufacturing steps of the test piece and b) the weld pass placement strategy of the test piece and c) that for the larger test piece previously neutron strain scanned reflecting a standard weld cap. Note the reduction in layers in b) but the same pass placement strategy as c).

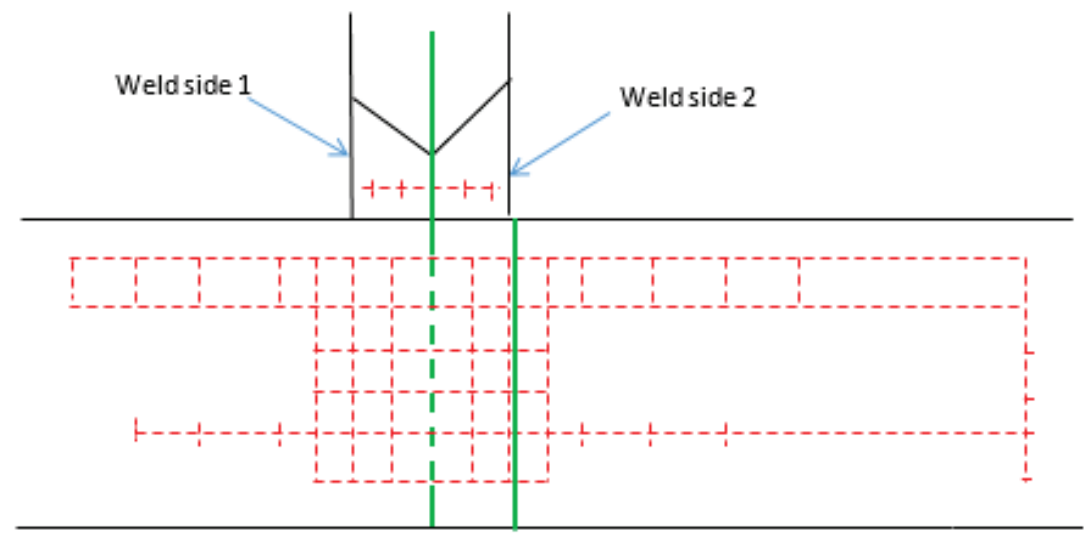

Fig. 2 Plan for residual stress measurements undertaken on $T$ butt weld test piece. The red grid represents the Neutron diffraction testing plan with strain measurements undertaken at the intersections of the transverse and vertical line. The two green lines are the locations of the centers of the DHD cores.

\section{Results}

The residual stress profiles determined by ND at the weld toes on side 1 and side 2, Fig. 3, are completely different as those on side 1 show a zigzag pattern with reduced magnitude close to the weld and then largely compressive at a depth of $10 \mathrm{~mm}$ below the concave surface before reverting to 
tensile at a depth below the concave surface of $20 \mathrm{~mm}$. The zigzag pattern for side 1 does not reflect that of a curved plate [2], [3] and therefore the original residual stress profile in the curved base plate has been over ridden. This is reinforced by the through thickness profiles for Side 2 in which there is no zigzag pattern and the 3 profiles follow the same pattern. For both side 1 and side 2 the profiles indicate that the residual stresses near the weld toe will be low tensile or low compressive stresses. This is supported by the result for the only location on the scan line across the weld where all 3 strains were captured was the one at side 2 of the weld pass closest to the weld cap surface. The residual stresses at this location were $-84.5 \mathrm{MPa},-160 \mathrm{MPa}$, and 19.1 $\mathrm{MPa}$ for the longitudinal transverse and normal directions respectively.
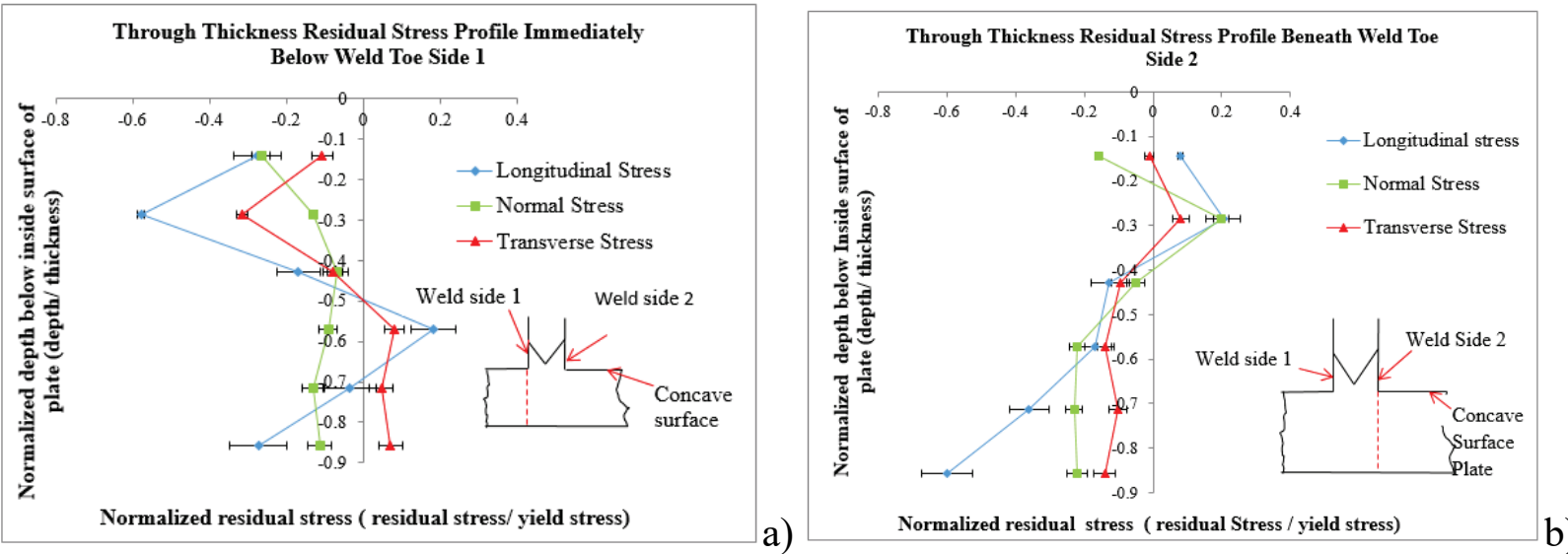

Fig 3 Through thickness plots of residual stress profiles in the base plate at the weld toes a) side 1 and $b)$ side 2 determined by ND

The through thickness profiles for the residual stresses determined by ND at the centerline of the web are presented in Fig. 4. They are radically different from the profiles presented in Fig. 3 in that they show substantial compressive residual stress values near mid thickness of the base plate changing to lower compressive values closer to the concave surface and to tensile values towards the convex surface. No residual stress results could be determined along this line in the weld and its heat affected zone, fusion zone and in the weld due to the beam path length in the longitudinal direction.

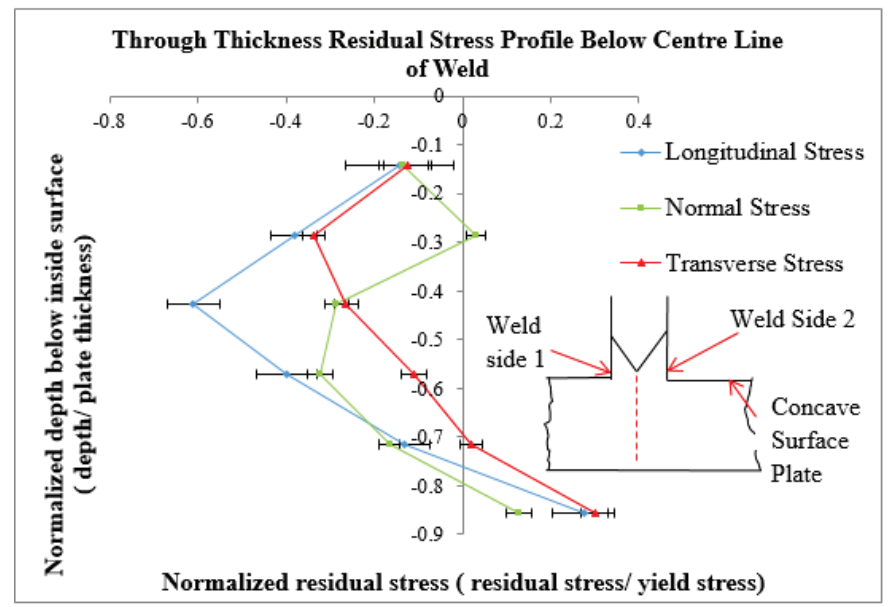

Fig. 4 Through thickness plots of residual stress profiles in the base plate at centerline of web determined by ND. 
The through thickness plots for the residual stress profiles obtained by iDHD/ DHD in the base plate and web are shown in Fig 5. The longitudinal and transverse residual stress profiles at the weld toe side 2 show a rapid reduction in the magnitude of the stress levels close to the weld on the concave side of the base plate, which is opposite to that for the un-welded base plate, and with a zigzag equilibrium

balancing pattern through thickness. Those through the centerline of the web while showing a zigzag pattern through the web indicated high tensile stresses in the weld and beginning of the web.

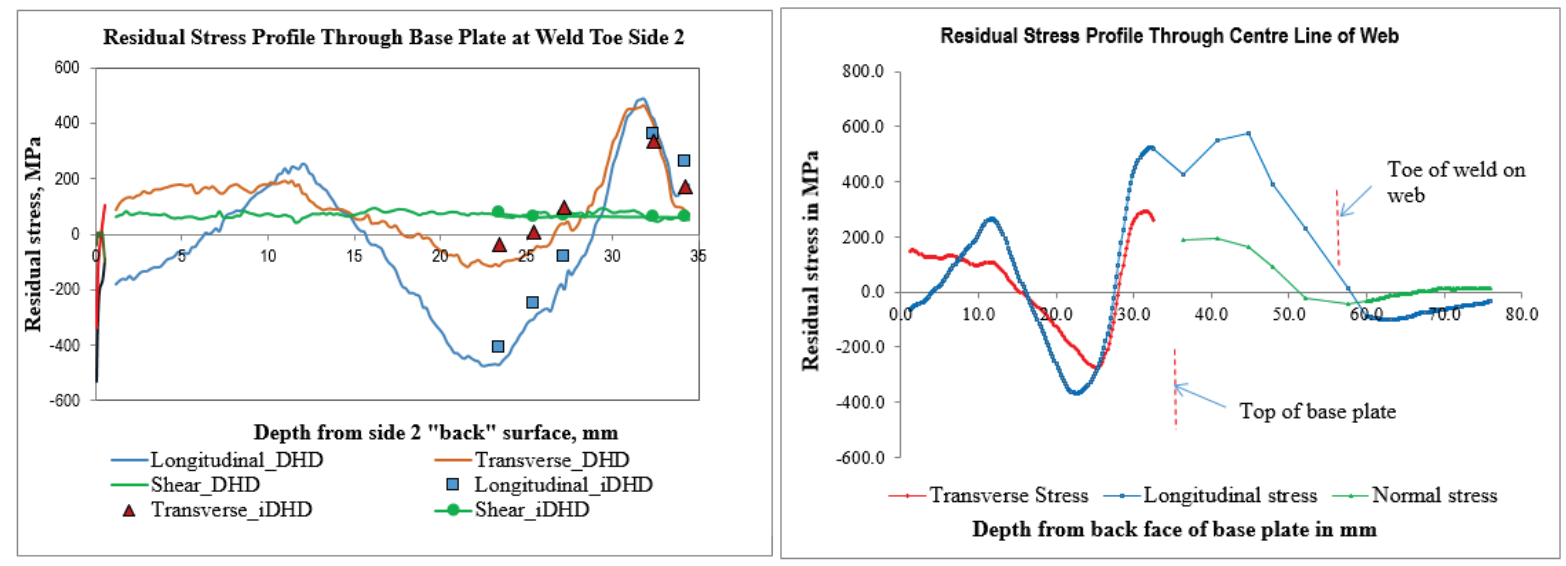

Fig. 5 Through thickness plots of the results of the DHD tests undertaken at a) the toe of the weld side 2 and b) through the centerline of the weld/web.

\section{Discussion}

The comparative plots of the through thickness residual stress profiles for ND and IDHD/DHD methods are presented in Figure 6. Looking first at those through the base plate and centerline of the web in Fig 6a) there appears to be good correlation for the results in that they are generally following the same profile though the ND results appear to be slightly out of phase with those determined by DHD. The differences reflect one of the limitations of ND in that due to time considerations for strain scanning, particularly for the longitudinal direction, and due to beam path length issues, no longitudinal strains were captured near the centerline in the weld. Since a more detailed profile was not achieved for the ND measurements there was a need to use alternative methods such as DHD/ iDHD.

Turning to the residual stress profiles at the weld toe region there is a distinct difference in the shapes of the profiles determined by ND for $10 \mathrm{~mm}$ from the centerline of the web i.e. directly under the weld toe to those determined by DHD centered $11 \mathrm{~mm}$ from the centerline of the web. There is closer correlation between the DHD profiles and the ND profiles measured $+15 \mathrm{~mm}$ from the centerline of the web both with respect to magnitudes of residual stress and to profile shape. The key contributing factors to this are the rapidly changing residual stresses induced by the welding moving away from the weld toe as well as the differences in gauge volume and position of the centers of gauge volume. The DHD is based on a $5 \mathrm{~mm}$ diameter core removed between $+8.5 \mathrm{~mm}$ and +13.5 $\mathrm{mm}$ whereas the ND measurements are based on a gauge volume from $+8 \mathrm{~mm}$ to $12 \mathrm{~mm}$ and hence the gauge volume for the ND is more closely aligned to the ND results centered on plus $15 \mathrm{~mm}$.

Notwithstanding this all the results at the weld toe indicate that there is a distinct drop in the magnitude of the residual stress profiles at the weld toe with the one ND result achieved in the weld cap indicating compressive residual stresses. It is apparent that equilibrium balancing through thickness results in the high tensile residual stress levels found in the weld and mid thickness of the 
base plate. As detailed in [3] the transverse strain profile across the weld/ web member $4 \mathrm{~mm}$ above the concave surface of the base plate varied from -681 micro strain in the weld cap side 2 to plateau at +1245 micro strain mid thickness before decreasing to -253 micro strain in the weld cap side 1 . The normal strain profile varied from 421 micro strain in the weld cap side 2 to reverse plateau at approximately zero micro strain mid thickness and then increase to 597 micro strain in the weld cap side 1. This strain profile supports what is evident in Fig 6. The reduction of the tensile residual stresses at the weld toes side 1 and side 2 indicates that the alternate pass placement strategy shown in Fig 1 will reduce the risk of HACC defects forming as well as SCC when in service. This is a major achievement as crack/ defect propagating from the weld toes through the base plate can lead to catastrophic failure of the structure. As crack propagation can lead to catastrophic failure the reduction in tensile residual stresses by the alternate pass placement strategy at the weld toes regions needs to be validated via further testing using an alternative method such as contouring the results of which are subject of a sister paper for this conference.
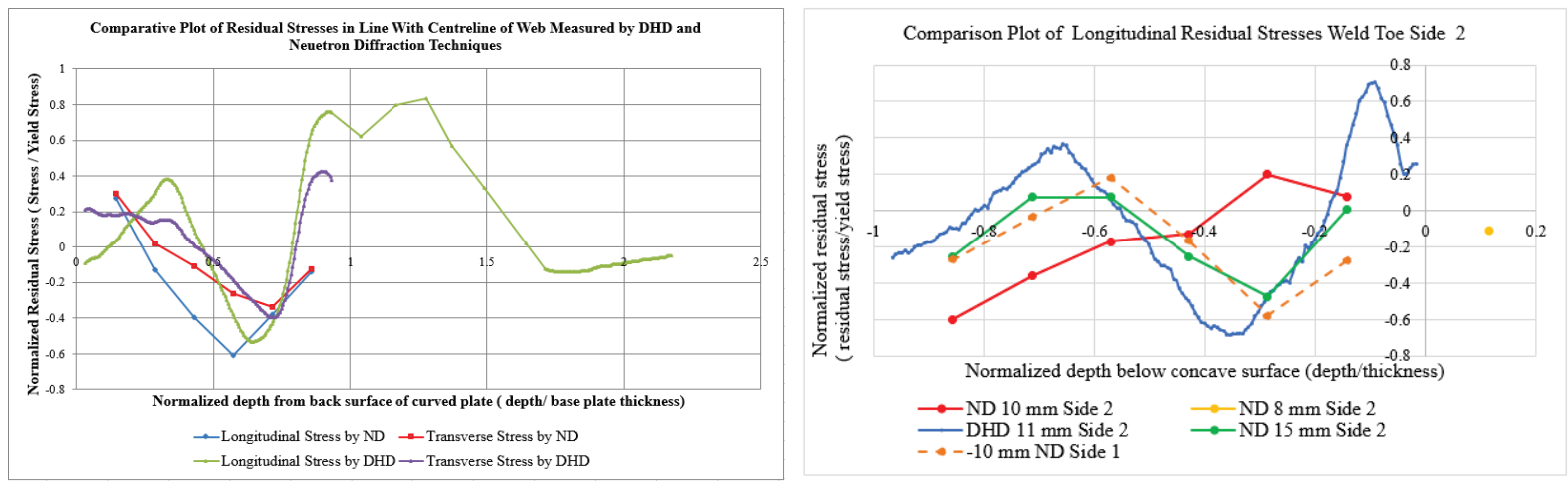

Fig 6. Comparative plots of the longitudinal residual stress profiles obtained by the ND and DHD at the centerline of the web on the left and at the weld toe side 2 on the right.

\section{Summary}

The residual stress profiles for the alternate pass placement strategy, from the ND and DHD testing undertaken on this test piece, clearly show that there is a reduction is the residual stress magnitude at the weld profile when compared with the yield strength values given in BS 7910. Further work needs to be undertaken by a $3^{\text {rd }}$ method such as contouring to establish the rate of change of the residual stress profiles away from the weld toe and to demonstrate the advantages of the alternate pass placement strategy of progressing weld passes from the base plate to the web to give confidence before implementing this strategy on full scale structures.

\section{References}

[1] BS 7910 "Guidance on methods of assessing the acceptability of flaws in metallic structures", 1999 with amendments 2000, 2009.

[2] S. Pearce "Breakdown of residual stresses in highly restrained thick section steel welds", Doctoral thesis, University of Adelaide 2009

[3] G. Sloan "The influence of welding variables on the residual stress profiles around $\mathrm{T}$ butt and MMAW butt welds in thick section high strength steel", Master's thesis, University of Adelaide 2015

[4] Information on http://www.veqter.co.uk 